\title{
Biosynthesis of Three Chalcone $\beta$-D-glucosides by Glycosyltransferase from Bacillus subtilis ATCC 6633
}

\author{
Yinuo Fei ${ }^{1}$, Yan Shao ${ }^{1}$, Weiwei Wang ${ }^{1}$, Yatian Cheng ${ }^{1}$, Boyang $\mathrm{Yu}^{1}$, Xiaorong $\mathrm{He}^{2 *}$, and Jian Zhang ${ }^{1,3 *}$ \\ ${ }^{1}$ State Key Laboratory of Natural Medicines, ${ }^{2}$ School of Engineering, China Pharmaceutical University, Nanjing 210009, P. R. China \\ ${ }^{3}$ ZhenPing Expert Workstation for Zhang Jian, Zhenping, Ankang, Shaanxi 725699, P. R. China
}

Received: February 25, 2021 / Revised: March 24, 2021 / Accepted: March 26, 2021

\begin{abstract}
Chalcones exhibit multiple biological activities. Various studies have attempted to modify the structure of chalcones with a special focus on the addition of substituents to the benzene rings. However, these chemical modifications did not improve the water solubility and bioavailability of chalcones. Glycosylation can markedly affect the physical and chemical properties of hydrophobic compounds. Here, we evaluated the ability of a highly promiscuous glycosyltransferase (GT) BsGT1 from Bacillus subtilis ATCC 6633 to biosynthesize chalcone glucosides. Purified BsGT1 catalyzed the conversion of 4'-hydroxychalcone (compound 1), 4'-hydroxy-4-methylchalcone (compound 2), and 4-hydroxy-4'-methoxychalcone (compound 3), into chalcone 4'-O- $\beta$-D-glucoside (compound 1a), 4-methylchalcone 4'-O- $\beta$-D-glucoside (compound 2a), and 4'methoxychalcone 4-O- $\beta$-D-glucoside (compound 3a), respectively. To avoid the addition of expensive uridine diphosphate glucose (UDP-Glc), a whole-cell biotransformation system was employed to provide a natural intracellular environment for in situ co-factor regeneration. The yields of compounds 1a, 2a, and 3a

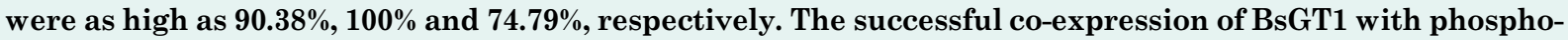
glucomutase (PGM) and UDP-Glc pyrophosphorylase (GalU), which are involved in the biosynthetic pathway of UDP-Glc, further improved the conversion rates of chalcones (the yields of compounds 1a and $3 a$ increased by approximately $10 \%$ ). In conclusion, we demonstrated an effective whole-cell biocatalytic system for the enzymatic biosynthesis of chalcone $\beta$-D-glucoside derivatives.
\end{abstract}

Keywords: Glycosyltransferase, Chalcone $\beta$-D-glucosides, Bacillus subtilis ATCC 6633, glycosylation

\section{Introduction}

Chalcones with the core scaffold of 1,3-diphenyl-2propen-1-ones are the precursors of flavanone, flavone, flavonol, anthocyanin, etc. They are widely distributed in licorice, safflower and other medicinal plants. The characteristic of three $\alpha, \beta$-unsaturated carbonyl systems connected to two aromatic rings makes them more flexible for a variety of targets [1]. At present, structural

\footnotetext{
*Corresponding authors

$X$. He

Tel: +86-25-86185730, Fax: +86-25-86185754

E-mail: hxrhf2659@163.com

J. Zhang

Tel: +86-25-86185157, Fax: +86-25-86185158

E-mail: 1020071849@cpu.edu.cn
}

modification of chalcone is mainly focused on the addition of substituents on the benzene rings. For instance, the addition of hydroxyl, methoxy and methyl could enhance its antimicrobial, anticancer and anti-inflammatory activity [2-4]. However, these modifications were not very helpful to improve the instability and poor water solubility of chalcones.

Glycosylation could greatly change the physical and chemical activities of hydrophobic compounds. These changes will not only affect the solubility and their chemical stability, but also affect intra- and intercellular transport of the molecular and biological half-life [5]. For example, Hesperidin methyl chalcone (HMC) is a kind of chalcone glycoside derivative, which has been proved to have anti-inflammatory activity in preclinical research [6]. Chalcone glycosylated derivatives are mainly syn- 
thesized by chemical methods [7]. Different from the tedious traditional chemical methods, biocatalytic glycosylation provides an environmentally friendly process and shows high stereoselectivity and regioselectivity [8].

In our previous study, BsGT1 from Bacillus subtilis ATCC 6633 was identified to catalyze the glycosylation of curcumin and analogs [9] and in this study three chalcone glycosides with methoxy, methyl, and hydroxyl groups substituted on the benzene ring were employed as the substrates to explore its catalytic capability on chalcones. Furthermore, we overexpressed two key enzymes, phosphoglucomutase (PGM) and uridine diphosphate glucose pyrophosphorylase (GalU), involved in the pathway of uridine diphosphate glucose (UDPGlc) synthesis in E. coli and successfully improved the efficiency of biosynthesis of chalcone glycosides by BsGT1 in the whole-cell system.

\section{Methods}

\section{Bacteria strains and culture conditions}

B. subtilis ATCC 6633 (a kind gift from the professor John P. N. Rosazza from University of Iowa), Escherichia coli DH5a (Novagen, USA) and E. coli BL21(DE3) (Transgen Biotech, China), which are the sources of BsGT1 gene (GenBank MK173044.1) and the host cells for cloning and gene expression, respectively, were cultured in $50 \mathrm{ml}$ of Luria-Bertani (LB) liquid medium. Kanamycin $(50 \mu \mathrm{g} / \mathrm{ml})$ was added if necessary.

\section{Cloning, expression and purification of BsGT1}

Using the genomic DNA of B. subtilis ATCC 6633 as a template, BsGT1 was amplified by PCR with the specific primers (shown in Table S1). The restriction sites of the primers were BamHI and XhoI, respectively. Then PCR products and pET-28a (+) (Novagen) were digested, linked, and transformed into E. coli BL21(DE3) to construct the recombinant strain. After induced by isopropyl- $\beta$-D-thiogalactopyranoside (IPTG, $0.5 \mathrm{mM}$ ) for $16 \mathrm{~h}\left(18^{\circ} \mathrm{C}, 200 \mathrm{rpm}\right)$, the cultured cells were collected and resuspended in $20 \mathrm{ml}$ lysis buffer $\left(50 \mathrm{mM} \mathrm{NaH}_{2} \mathrm{PO}_{4}\right.$, $300 \mathrm{mM} \mathrm{NaCl}, \mathrm{pH}$ 8.0). Then, the cells were disrupted using a sonicator on ice. The supernatant was applied to a pre-equilibrated Ni-NTA column and eluted by elution buffer containing a 10-250 mM imidazole gradient. The target protein was eluted by an elution buffer containing
$50 \mathrm{mM}$ imidazole. The elution buffer containing the target protein was concentrated to $1 \mathrm{ml}$ as a purified enzyme by $15 \mathrm{ml} 10 \mathrm{kDa}$ MWCO filters (EMD, Millipore).

\section{Catalytic activity assay}

The catalytic activity of the BsGT1 to the three chalcone glycosides was verified by $300 \mu \mathrm{l}$ reaction buffer containing $5 \mu \mathrm{g}$ of purified protein, $75 \mu \mathrm{M}$ Tris$\mathrm{HCl} \mathrm{pH} \mathrm{8.0,} 36.94 \mu \mathrm{M}$ UDP-Glc and $0.84 \mathrm{mM}$ substrates (dissolved in DMSO). The reactions were incubated at $37^{\circ} \mathrm{C}$ for $3 \mathrm{~h}$ and extracted three times with equal volume ethyl acetate, and the organic phase was dried and added $200 \mu \mathrm{l}$ methanol for analysis by HPLC and HPLC-QTOF-MS (Agilent Technologies, USA) at UV $254 \mathrm{~nm}$ with a reverse-phase C18 column (4.6 $\times$ $250 \mathrm{~mm}, 5 \mu \mathrm{m}$ particles; Welch, China).

\section{NMR analysis}

The substrates and glycosylated products were separated by dichloromethane-methanol (40:1 and 9:1, v/v). After separation from the silica gel column, the structures of glycosylated products were determined by ${ }^{1} \mathrm{H}$ NMR and ${ }^{13} \mathrm{C}-\mathrm{NMR}$ with DMSO- $d 6$ as solvent. And the ${ }^{1} \mathrm{H}-\mathrm{NMR}$ and ${ }^{13} \mathrm{C}-\mathrm{NMR}$ spectra were obtained by Bruker AV-600 spectrometer (Bruker, USA).

\section{Co-expression of BsGT1, GalU, PGM and whole-cell bio- transformation}

galU (GenBank CP010816.1 17836-18785) and pgm (GenBank CP010816.1 9622-11287) were amplified from E. coli BL21(DE3) genomic DNA by PCR. Vector pACYCDuet-1 (Novagen) and pCDFDuet-1 (Novagen) were selected to harbor pgm and galU. Pgm was cloned between BamHI and PstI, and galU was subsequently cloned between XhoI and AvrII generating plasmid pACYCDuet-1-PGM-GalU and pCDFDuet-1-PGM-GalU. Two recombinant plasmids were extracted and transformed into the recombinant $E$. coli BL21(DE3) containing BsGT1, respectively. The recombinant strains BL21BsGT1-pA-PGM-GalU and BL21-BsGT1-pC-PGM-GalU were prepared and cultured at $37^{\circ} \mathrm{C}, 220 \mathrm{rpm}$ for $6 \mathrm{~h}$, respectively. When the $\mathrm{OD}_{600}$ reached $0.6,0.5 \mathrm{mM}$ IPTG was added. After induced for $16 \mathrm{~h}$, the glucose $(0.1 \mathrm{M})$ and the substrates $(0.84 \mathrm{mM})$ were added for biotransformation of $24 \mathrm{~h}$ at $37^{\circ} \mathrm{C}, 200 \mathrm{rpm}$. Finally, equal volume methanol was added to stop the reactions and then 
analyzed with HPLC, and these experiments were repeated three times.

\section{Results}

\section{Expression, purification and SDS-PAGE analysis of BsGT1}

The glycosyltransferase BsGT1 was expressed in $E$. coli BL21(DE3), purified by Ni-NTA and assessed molecular weight via SDS-PAGE. SDS-PAGE analysis showed that the molecular weight of the protein was about $44 \mathrm{kDa}$ (Fig. 1) determined by protein marker, which was the same as our previous verification [9].

\section{Biotransformation of chalcones by the purified BsGT1}

The reactions were conducted with $75 \mu \mathrm{M}$ Tris- $\mathrm{HCl} \mathrm{pH}$ 8.0, $36.94 \mu \mathrm{M}$ UDP-Glc and $0.84 \mathrm{mM}$ substrates and were preliminarily detected by HPLC to verify the glycosylation activity of BsGT1 towards the three chalcone glycosides. All the mixtures displayed a new peak with shortened retention time than that of substrates. To further determine whether glycosylation occurred, HPLC-QTOF-MS analysis was conducted and the results showed that $162 \mathrm{Da}$ was added to $1 \mathbf{a}\left([\mathrm{M}-\mathrm{H}]^{-}, m / z\right.$ $=\sim 385.1572$ ) (Fig. 2a), 2a ([M-H] $\left.]^{-}, m / z=\sim 399.1740\right)$

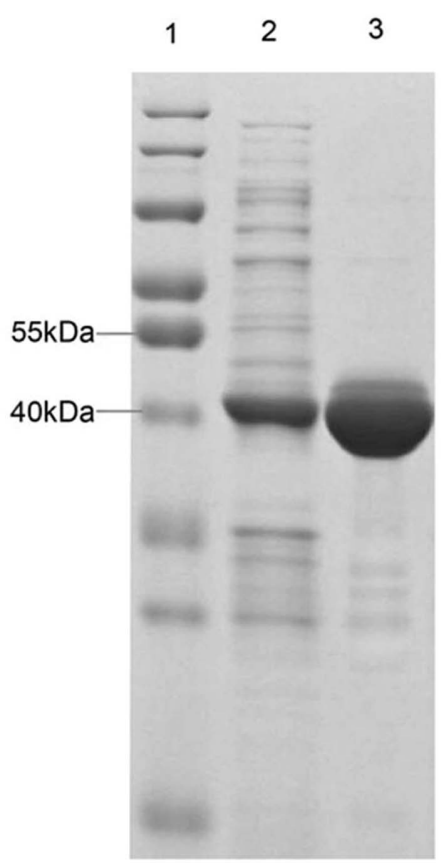

Fig. 1. Expression, purification, and analysis of BsGT1. Lane 1, protein marker; lane 2, induction of BsGT1 protein; lane 3, purified BsGT1.

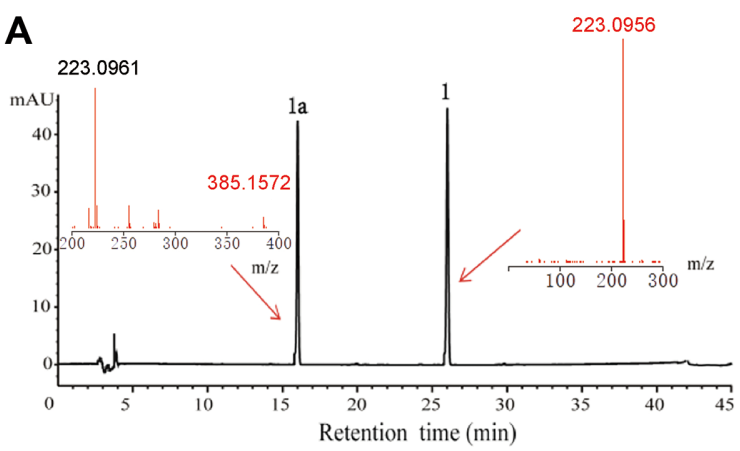

B
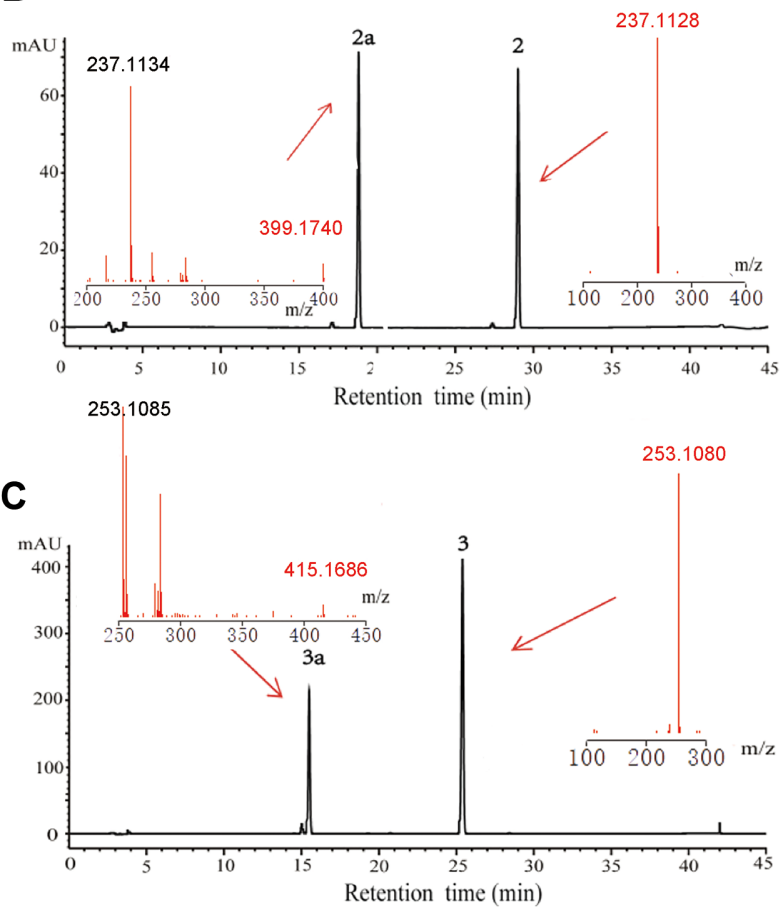

Fig. 2. HPLC and QTOF-MS analysis. HPLC analysis of the glycosylation activity of BsGT1 catalyzing Compound 1 (A), Compound 2 (B) and Compound 3 (C), and QTOF-MS analysis of those catalyzed by BsGT1 (red numbers are molecular ion peaks $[\mathrm{M}-\mathrm{H}]^{-}$of glucoside derivatives and substrates).

(Fig. 2b), and 3a ([M-H] $]^{-}, m / z=\sim 415.1686$ ) (Fig. 2c) compared with Compound $\mathbf{1}\left([\mathrm{M}-\mathrm{H}]^{-}, m / z=\sim 223.0956\right)$, 2 ([M-H] $\left.]^{-}, m / z=\sim 237.1128\right)$, and $3\left([\mathrm{M}-\mathrm{H}]^{-}, m / z=\right.$ $\sim 253.1080$ ), respectively. All of these indicated that three chalcone substrates may undergo glycosylation to produce monoglucosides by BsGT1.

\section{Structural elucidation of three chalcone glucosides}

The purified glucosylated products were dissolved in DMSO- $d 6$ and analyzed by Bruker AV-600 spectrometer 
<smiles>O=C(C=Cc1ccccc1)c1ccc(O)cc1</smiles>

1<smiles>Cc1ccc(/C=C/C(=O)c2ccc(O)cc2)cc1</smiles>

2<smiles>COc1ccc(C(=O)/C=C/c2ccc(O)cc2)cc1</smiles>

3

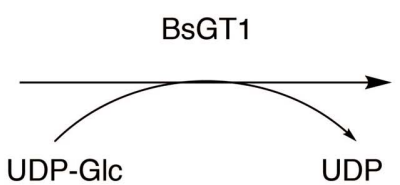<smiles>O=C(/C=C/c1ccccc1)c1ccc(OC2OC3C(O)C(O)C(O)C(O)C3O2)cc1</smiles>

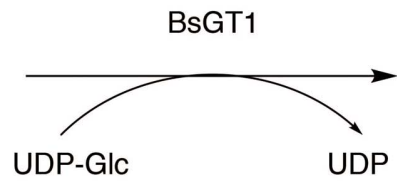<smiles>Cc1ccc(/C=C/C(=O)c2ccc(OC3OC4COC(O)C(CO)C3OC4O)cc2)cc1</smiles>

$2 a$<smiles>COc1ccc(C(=O)/C=C/c2ccc(OC3OC4C(O)C(O)C(O)C3C4(Br)Br)cc2)cc1</smiles>

Fig. 3. Schematic representation of the glycosylation of three chalcones by BsGT1.

for ${ }^{1} \mathrm{H}-\mathrm{NMR}$ and ${ }^{13} \mathrm{C}-\mathrm{NMR}$ (Figs. S1-3 and Table 4). The ${ }^{13} \mathrm{C}-\mathrm{NMR}$ spectrum of $\mathbf{1 a}$ showed characteristic carbon signals of glucose moiety at $\delta \mathrm{c} 100.21,77.64,77.00$, 73.62, 70.06, 61.05, and the signal for C-4' which shifted up-field by $1.62 \mathrm{ppm}$ indicated that the new glycosylated group located at C-4'. In the ${ }^{1} \mathrm{H}-\mathrm{NMR}$ spectrum, signals at $\delta_{\mathrm{H}} 5.05(\mathrm{~d}, J=7.4 \mathrm{~Hz}, 1 \mathrm{H})$ and $3.17-3.72(6 \mathrm{H})$ were obtained, which confirmed the attachment of one glucosyl moiety with configuration. Similarly, in the ${ }^{1} \mathrm{H}-\mathrm{NMR}$ spectrum of $\mathbf{2 a}$ and $\mathbf{3 a}$, the anomeric proton signals at $\delta_{\mathrm{H}} 5.05(\mathrm{~d}, J=7.4 \mathrm{~Hz}, 1 \mathrm{H})$ and $4.97(\mathrm{~d}, J=7.5 \mathrm{~Hz}, 1 \mathrm{H})$, respectively indicated the $\beta$-configuration of $\mathbf{2 a}$ and $\mathbf{3 a}$. These results demonstrated that compound 1a, $\mathbf{2 a}$ and $3 \mathbf{a}$ were characterized as chalcone 4'-O- $\beta$-D-glucoside, 4methylchalcone 4'-O- $\beta$-D-glucoside and 4'-methoxychalcone 4-O- $\beta$-D-glucoside, respectively (Fig. 3).

\section{Co-expression of BsGT1, GalU, and PGM}

The whole-cell catalysis is a more practical method compared with pure enzyme catalysis, because the latter requires expensive UDP-Glc and there are also many steps in purifying enzymes. PGM and GalU could be overexpressed to increase the carbon flux toward UDPGlc synthesis at the branch point of glucose-6-phosphate (G6P) by catalyzing the conversion of G6P to glucose-1phosphate (G1P) and the formation of UDP-Glc from G1P and uridine triphosphate (UTP), respectively [10].
In order to get glucosides more efficiently, two vectorspACYCDuet-1 and pCDFDuet-1-with different copies but both compatible with pET28a $(+)$ were selected to harbor pgm and galU, and then a co-expression system of PGM, GalU and BsGT1 was established for a wholecell bioconversion reaction (Fig. 4). Then the analysis of SDS-PAGE was carried out and the result showed that PGM, GalU and BsGT1 exhibited single bands corresponding to 60, 32 and $44 \mathrm{kDa}$, respectively (Fig. 5).

\section{Biotransformation of chalcones by recombinant $E$. coli}

When the final concentration of added substrates reached $0.84 \mathrm{mM}$, the relative conversion rate of Compound 1, 2 and 3 by BL21-BsGT1 was $90.37 \%$, $100 \%$ and $74.79 \%$ (Fig. 6), respectively. Two constructed recombinant strains BL21-BsGT1-pA-PGM-GalU, and BL21-BsGT1-pC-PGM-GalU exhibited the improved production of chalcone glucosides, approximately $10 \%$ higher for both 1a and $\mathbf{3 a}$, in comparison with the control strains which only contained BsGT1.

\section{Discussion}

Chalcones are one type of leading compounds with multiple biological activities such as anti-inflammatory, anti-cancer and anti-gout [11], and its derivatives have been widely used in clinic, like metochalcone and sofalcon. 

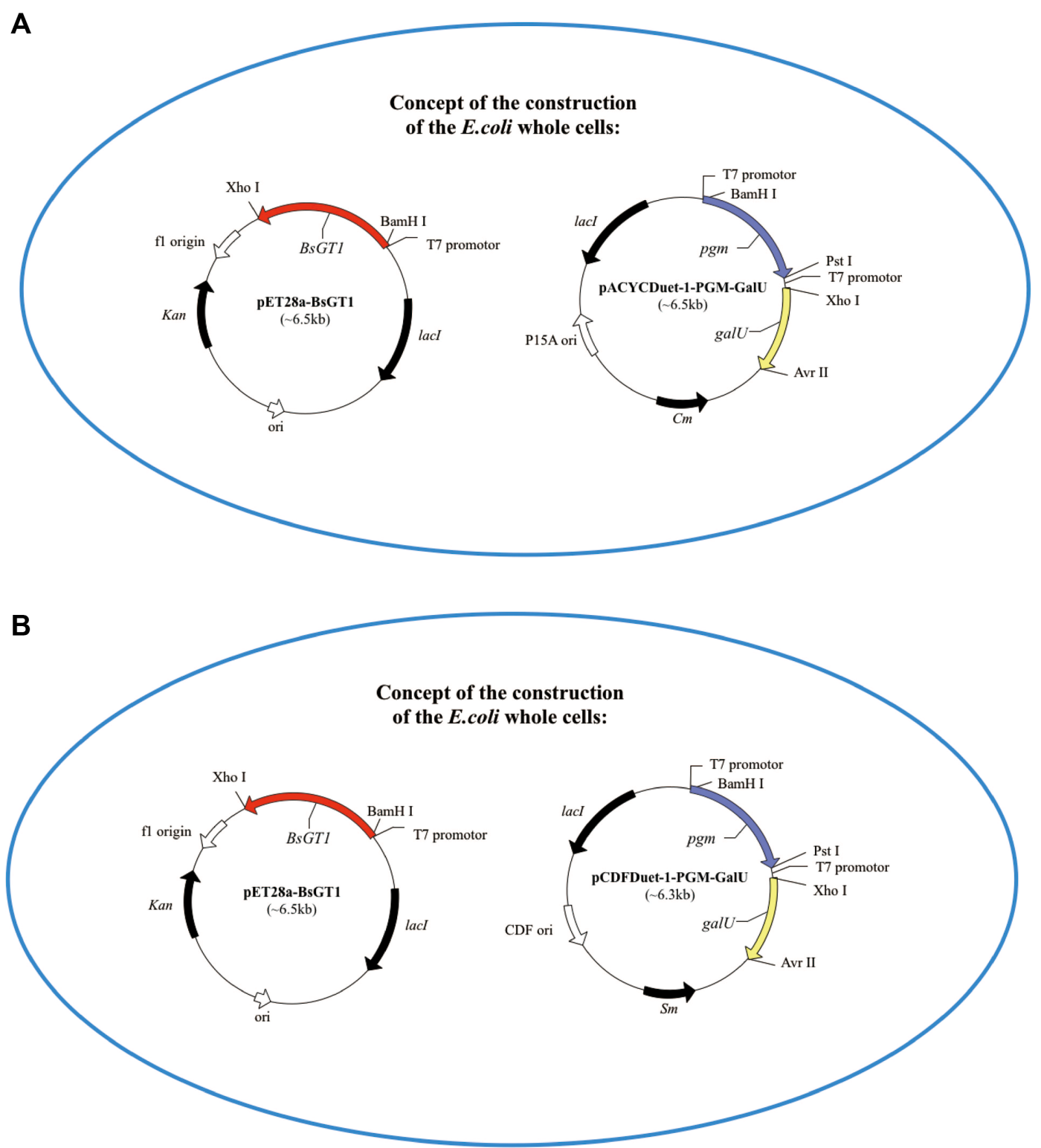

Fig. 4. Design of the recombinant whole-cell catalyst. (A) Design of the recombinant whole-cell catalyst containing BsGT1 from B. subtilis ATCC 6633 in a pET-28a (+) vector and pgm/galU in a pACYCDuet-1 vector; (B) Design of the recombinant whole-cell catalyst containing BsGT1 in a pET-28a (+) vector and pgm/galU in a pCDFDuet-1 vector.

However, low water solubility and bioavailability of chalcones challenge their clinical development. Therefore, the synthesis of chalcone derivatives is of great significance for clinical application. It is widely believed that the glycosyl modified molecules often show the ability to improve stability and water-solubility, reduce the toxicity and enhance the interaction between the compound and other cell components [12]. In this work, three tested chalcones, 4'-hydroxychalcone (Compound
1), 4'-hydroxy-4-methylchalcone (Compound 2) and 4hydroxy-4'-methoxychalcone (Compound 3) were all converted to their corresponding $\beta$-D-glucosides, 1a, $2 \mathbf{a}$ and 3a. This rapid preparation method of chalcone glucoside derivatives we proposed provides the basis for the discovery of chalcone derivatives with better clinical value. On the other hand, the discovery of the potential pharmacological activity of these three chalcone glucosides becomes possible. 


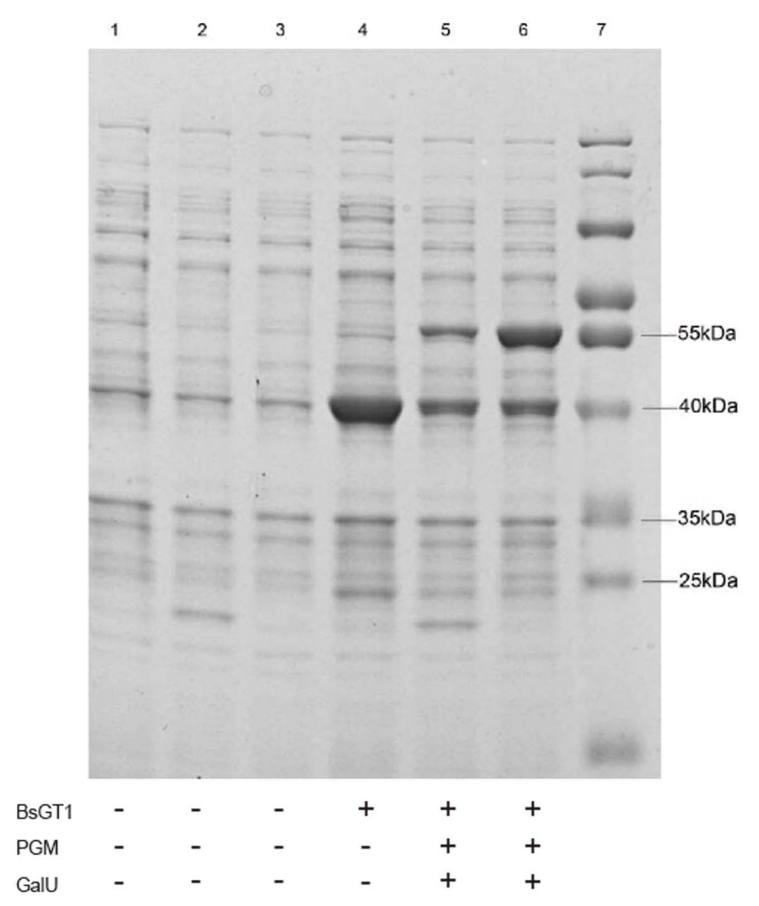

Fig. 5. Analysis of expression levels of BsGT1, PGM, GalU by SDS-PAGE. Lane 1, cleavage products of $E$. coli with empty plasmid pET-28a (+); lane 2, cleavage products of $E$. coli with plasmid pET-28a (+) and pACYCDuet-1; lane 3, cleavage products of $E$. coli with plasmid pET-28a (+) and pCDFDuet-1; lane 4, induction of recombinant soluble BsGT1 protein; lane 5 , induction of recombinant soluble BL21-BsGT1-pA-PGM-GalU protein; lane 6, induction of recombinant soluble BL21-BsGT1-pC-PGMGalU protein; lane 7, protein marker.

Compared with GTs from plants, microbial GTs generally exhibit remarkable substrate universality [13, 14]. Our previous study has proved that BsGT1 from $B$. subtilis ATCC 6633 had excellent glycosylation ability toward curcumin and analogs [9]. The glycosylation of three chalcone derivatives demonstrated its substrate promiscuity again. What's more, the phenol hydroxyl groups in either rings A or B of chalcone could be glycosylated, and the substitution of methyl or methoxy groups in the benzene ring did not affect the glycosylation of BsGT1. Therefore, BsGT1 could be used as a robust biocatalyst for glycosylation of natural and unnatural products for new drug discovery.

Enzyme catalysis and whole-cell catalysis are the two main methods to obtain glucosides by GTs. Enzyme catalysis has the advantages of fast reaction and high

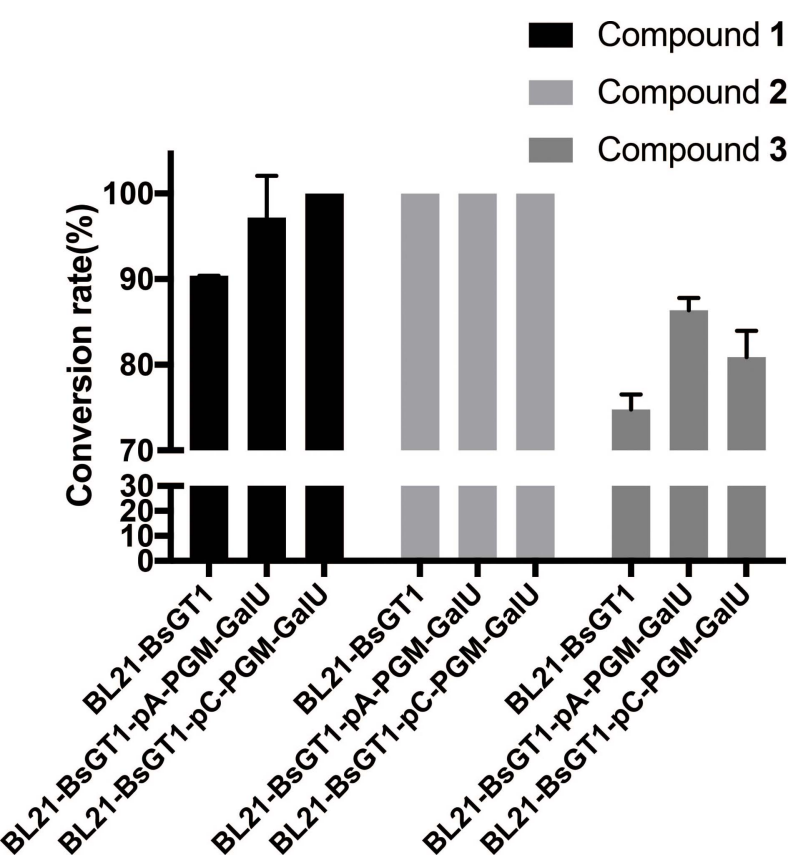

Fig. 6. Whole-cell biotransformation of three chalcones by BL21-BsGT1, BL21-BsGT1-pA-PGM-GalU, and BL21-BsGT1PC-PGM-GalU.

specificity. However, the purification process of the enzyme is complex and the cost of adding activated glucosyl donor is high. The availability of UDP-Glc could be improved by overexpressing PGM and GalU in recombinant $E$. coli, which has been developed to greatly increase the production rate of pelargonidin 3-O-glucoside and cyanidin 3-O-glucoside [15]. In our study, the coexpression of PGM, GalU, and BsGT1 using compatible vectors in $E$. coli BL21(DE3) could increase the relative conversion rate of $0.84 \mathrm{mM}$ chalcones up to $100 \%$, which provides a way to synthesize chalcone glucosides in high efficiency. In addition, due to high promiscuity of BsGT1, this co-expression system could be extended to glycosylation of more natural and unnatural compounds with different structures.

In conclusion, three chalcone glucosides were biosynthesized by BsGT1 in this study. It is a practical method to enhance the glycosylation by the co-expression system of PGM, GalU, and BsGT1, which can obtain the chalcone $\beta$-D-glucosides simply and quickly. Based on the extensive pharmacological activities and low toxicity of chalcones, further research on chalcone glucosides will develop more new lead compounds for clinical application. 


\section{Acknowledgments}

This work was supported by National Nature Science Foundation of China (NSFC NO. 21302052) and the "Program for New Century Excellent Talents in University" awarded to Prof. Jian Zhang (NECT-11-0739). Thanks also give to Postgraduate Research \& Practice Innovation Program of Jiangsu Province (SJKY19_0658).

\section{Conflict of Interest}

The authors have no financial conflicts of interest to declare.

\section{References}

1. Orlikova B, Tasdemir D, Golais F, Dicato M, Diederich M. 2011. Dietary chalcones with chemopreventive and chemotherapeutic potential. Genes Nutr. 6: 125-147.

2. Al-Mamary M, Al-Mikhlafi SA, Jaadan B. 2014. Synthesis and biological activities of some chalcone derivatives. Int. J. Chem. Pharm. Sci. 5: 8.

3. Ma Y, He Y, Yin T, Chen H, Gao S, Chalcone Derivatives M. 2018. Metabolism of phenolic compounds in LPS-stimulated Raw264.7 Cells can impact their anti-inflammatory efficacy: Indication of hesperetin. J. Agric. Food Chem. 66: 6042-6052.

4. Sangpheak K, Mueller M, Darai N, Wolschann P, Suwattanasophon C, Ruga R, et al. 2019. Computational screening of chalcones acting against topoisomerase Il $\alpha$ and their cytotoxicity towards cancer cell lines. J. Enzyme Inhib. Med. Chem. 34: 134-143.

5. Hofer B. 2016. Recent developments in the enzymatic O-glycosylation of flavonoids. Appl. Microbiol. Biotechnol. 100: 42694281.
6. Pinho-Ribeiro FA, Hohmann MSN, Borghi SM, Zarpelon AC, Guazelli CFS, Manchope MF, et al. 2015. Protective effects of the flavonoid hesperidin methyl chalcone in inflammation and pain in mice: Role of TRPV1, oxidative stress, cytokines and NF-KB. Chem. Biol. Interact. 228: 88-99.

7. Manna T, Pala K, Janaa K, Misraa AK. 2019. Anti-cancer potential of novel glycosylated 1,4-substituted triazolylchalcone derivatives. Bioorg. Med. Chem. Lett. 29: 126615.

8. Xiao J, Muzashvili TS, Georgiev Ml. 2014. Advances in the biotechnological glycosylation of valuable flavonoids. Biotechnol. Adv. 32: 1145-1156.

9. Cheng Y, Zhang J, Shao Y, Xu Y, Ge H, Yu B, et al. 2019. Enzymecatalyzed glycosylation of curcumin and its analogues by glycosyltransferases from Bacillus subtilis ATCC 6633. Catalysts 9: 734.

10. Mao Z, Shin H-D, Chen RR. 2006. Engineering the E. coli UDP-Glucose synthesis pathway for oligosaccharide synthesis. Biotechnol. Prog. 22: 369-374.

11. Gomes MN, Muratov EN, Pereira M, Peixoto JC, Rosseto LP, Cravo PVL, et al. 2017. Chalcone derivatives: Promising starting points for drug design. Molecules 22: 1210.

12. Lowery JJ, Yeomans L, Keyari CM, Davis P, Porreca F, Knapp BI, et al. 2007. Glycosylation improves the central effects of DAMGO. Chem. Biol. Drug Des. 69: 41-47.

13. Pandey RP, Bashyal P, Parajuli P, Yamaguchi T, Sohng JK. 2019. Two Trifunctional Leloir Glycosyltransferases as biocatalysts for natural products glycodiversification. Org. Lett. 21: 8058-8064.

14. Dai L, Li J, Yao P, Zhu Y, Men Y, Zeng Y, et al. 2017. Exploiting the aglycon promiscuity of glycosyltransferase Bs-YjiC from Bacillus subtilis and its application in synthesis of glycosides. J. Biotechnol. 248: 69-76.

15. Yan Y, Li Z, Koffas MAG. 2008. High-yield anthocyanin biosynthesis in engineered Escherichia coli. Biotechnol. Bioeng. 100: 126-140. 